\title{
UAV PHOTOGRAMMETRY TO MONITOR DYKES - CALIBRATION AND COMPARISON TO TERRESTRIAL LIDAR
}

\author{
V.Tournadre ${ }^{\mathrm{a} *}$, M.Pierrot-Deseilligny ${ }^{\mathrm{b}}$, P.H.Faure ${ }^{\mathrm{c}}$ \\ ${ }^{a}$ IGN-ENSG, Institut Géographique National, 77420 Champs-sur-Marne, France - vincent.tournadre@ensg.eu \\ b IGN-ENSG, Institut Géographique National, 77420 Champs-sur-Marne, France - marc.pierrot-deseilligny@ensg.eu \\ c CNR-CACOH, Compagnie Nationale du Rhône, 69000 Lyon, France - p.faure@cnr.tm.fr
}

KEY WORDS: RPAS, Dyke, Metrology, Monitoring, Drift effect, MicMac

\begin{abstract}
:
Unmanned Aerial Vehicles (UAV) and photogrammetry are two fields that have been boosted these last years. Using aerial means, one can easily acquire aerial data and produce high resolution dense surface models, orthophotos,...

IGN (the French Mapping Agency) and CNR (Compagnie Nationale du Rhône, which is the concessionary of the Rhône river and a hydraulic energy producer) have associated themselves on a thesis protect. The aim is to be able to monitor dykes from images acquired by UAV and take benefit from their convenience, targeting a centimetric accuracy on the Z-axis. This article presents our motivations and the problems we have faced in our first experiments. We also worked on a site covered by a terrestrial Lidar survey, and studied how minimizing the bundle adjustment residuals by using different calibrations would influence the quality of the computed models. Finally, we will introduce in a last part our last experiments to get a better understanding of poses estimation accuracy.
\end{abstract}

\section{INTRODUCTION}

Unmanned Aerial Vehicle (UAV), Remotely Piloted Automated Systems (RPAS), Drones,... all these words refer to the same object. People used to see them as a tool to drop bombs, but now the civilian world is getting its hands on it. From precision crop management to beer delivery during music festival, many applications have been explored. As a hydraulic energy producer, the Compagnie Nationale du Rhône (CNR) is more interested in a system that could help them to monitor their dykes.

A dyke is a very important component of a river. It has to create a reservoir upstream the hydro power, and it has to be solid enough to endure the years. Even though we don't usually notice it, some of them are slowly sinking. Considering the major issues in case of a breach and the french regulation, they have to be regularly inspected and monitored.

CNR has set up a network of topographic references through a precision levelling process all along the river, from the Switzerland border to the Mediterranean sea (over $400 \mathrm{~km}$ ). Four offices specialised in land surveys have been dispatched along, monitoring structures all year round.

This organisation has been sufficient to avoid any major problem, but we think that it is possible to use UAV combined with the most recent photogrammetric tools to get a complementary system. The idea is to end up in a system that would be cheaper, faster, more accurate and with a better resolution than traditional surveying methods.

In that case, UAV are interesting as they offer a cheap alternative to aircrafts or helicopters. They also fly closer to the ground, and so have the capability to acquire pictures with centimetric or even smaller pixels.

Two kinds of acquisitions are considered :

- Regular acquisitions - once a year - on sensitive sites, aiming to detect centimetric deformations on dykes measuring up to $1000 \mathrm{~m}$ long ;

- Exceptional acquisitions, aiming to deliver rapidly some comparison with an initial state in case of a crisis situation (earthquake, flooding, sailing accident). The length would depend on the situation, but CNR would like to get at least $5 \mathrm{~cm}$ accuracies in such cases.

Using UAV for mapping purposes is nothing new. Many companies offer such services in France. However, most of the people in the UAV community don't have any background in photogrammetry, and to our knowledge, none is able to produce DSM with a centimetric accuracy. IGN has the intention to fill that gap and enter in the UAV market, as well as well as most European national mapping agencies (Cramer 2013).

This article presents our first experiments on two test sites, and the challenges we have faced to reach the expected precision. The first one has led us to think that there is a general drift effect in the photogrammetric process. On the second site, a previous Lidar survey has been used to finely compare both technologies, and to get a better understanding of the drift. Finally, we will introduce some of our last work, where we simulated a UAV flight at a scale of $1 / 20$, and measured the positions of the cameras with a micronic accuracy. Note that all the photogrammetric processes have been run with the open source suite Pastis-Apero-MicMac developed at IGN.

\section{FIRST EXPERIMENTS}

\subsection{Previous work at La Pallière}

On August 2011, a first UAV flight had been conducted over a small portion of La Pallière's dyke. Part of another thesis project, it was aiming to get a first tangible idea on how to organize such an operation, and the difficulties that could be faced.

If theoretically, using UAV for photogrammetric purposes might seem simple, some complications often happen on the field. The team which conducted this survey faced some of them. On the first flights, they did not put adhesive tape on the lense, which was a zoom, so that the focal length changed all along the photographic survey. They also used a bad SD card reader, which damaged the memory card and almost led the lost of the pictures. 


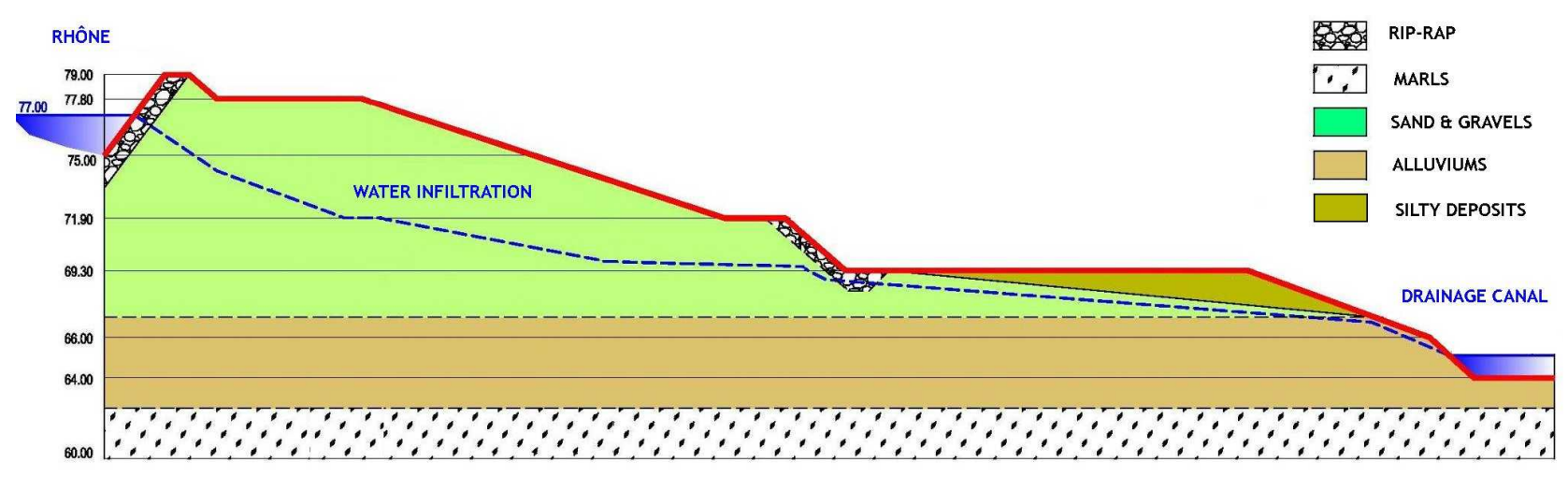

Higure 1 : Iransversal section ot a dyke

They were however able to produce high quality pictures of a 500 meters section. The data treatment has been complicated ; some first miscomputations of a proper calibration model delivered a curved model. Further work on the images delivered better results, and could have led to conclude that the technology is efficient, but they were lacking of data to be able to certify any kind of precision.

The idea of a thesis dedicated to that problematic started here.

\subsection{Beginning of the thesis}

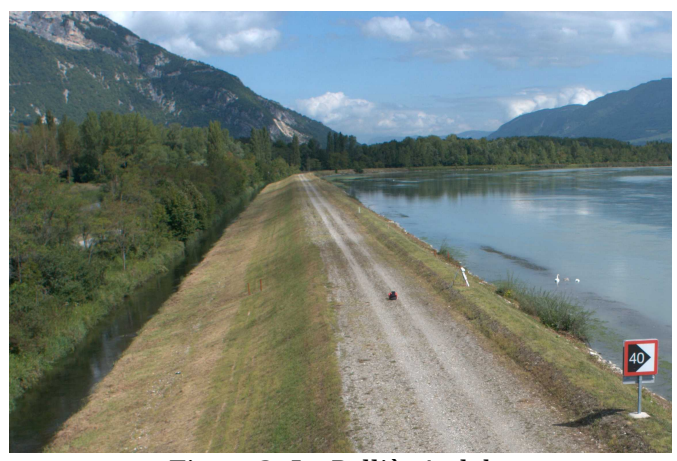

Figure 2: La Pallière's dyke

La Pallière's dyke is one of our test site, on which we will conduct regular acquisitions. This $1100 \mathrm{~m}$ section is interesting because previous land surveys have shown for many years that it is not perfectly stable.

As a first test site, we set up 50 ground control points (GCP) to be sure we have enough data to analyse the results. As we are concerned about the accuracy on the Z-axis, we surveyed those points using precision levelling techniques, while XY were determined by RTK GPS.

Setting up points that way is time consuming, as two days were needed to complete the full operation. Even though some improvements are expected very soon to embark RTK GPS on UAV (Stempfhuber 2013, Piksi 2013), we do not think it will yet be accurate enough on the altitude, and so a precision levelling might still be necessary in the future for that range of accuracies. An important part of the thesis will be to work on how to reduce the amount of GCP needed.

The UAV used for that mission was an HexaXL (MikroKopter) flew by a private company providing photographic acquisition services. We used two cameras :

- a Sony NEX7, including a 24 MP APS-C sensor, equipped with a Carl Zeiss $24 \mathrm{~mm}$ f/1.8 fixed lens (equivalent to $35 \mathrm{~mm}$ ), which is the flight company's property

- $\quad$ a Sony RX1 compact camera, with a 24MP full-frame sensor, on which a $35 \mathrm{~mm}$ Carl Zeiss $\mathrm{f} / 2.0$ lens is sealed, which has been acquired by IGN
The RX1 produces much better images, with a very good sharpness, very low distortion and no noticeable chromatic aberrations. Its global shutter also guarantees that every pixel is recorded at the same time. On the other hand, the NEX7 is popular among the community, so we were interested in benchmarking it.

We defined the flight plan before the mission, forecasting to fly at $60 \mathrm{~m}$ to get pictures sizing $40 \times 60 \mathrm{~m}$, each pixel covering about $1 \mathrm{~cm}^{2}$. Waypoints were set each 8 meters along the 1100 meters dyke on two parallel strips (with a $12 \mathrm{~m}$ gap) to get a global $80 \%$ overlap. Due to legal constraints, pilots had to navigate their RPAS from no more than $150 \mathrm{~m}$, so the flight plan had been accordingly cut in small portions.

\subsection{Photogrammetric survey}

On the first day, we mounted the NEX7 on a gyro stabilized gimbal to counter any vibration. That did not work pretty well as the vibrations propagated alongside the gimbal. Comparable results had been obtained by using the RX1.

On the second day, we fixed the problem by using a piece of foam between the structure and the cameras. The vibration effect disappeared, but we still had some bad image quality, especially in the corners (chromatic aberration, high distortion,...).

Testing the NEX7 at the ground showed the same kind of aberrations. Having used some other NEX7 after that mission, we think that the camera lens might had an imperfection. So we did not actually used those pictures, but covered the flight with the RX1 on a third day. 215 images have been acquired after two hours of a very stable flight, delivering high quality pictures.

\subsection{Photogrammetric processing and results}

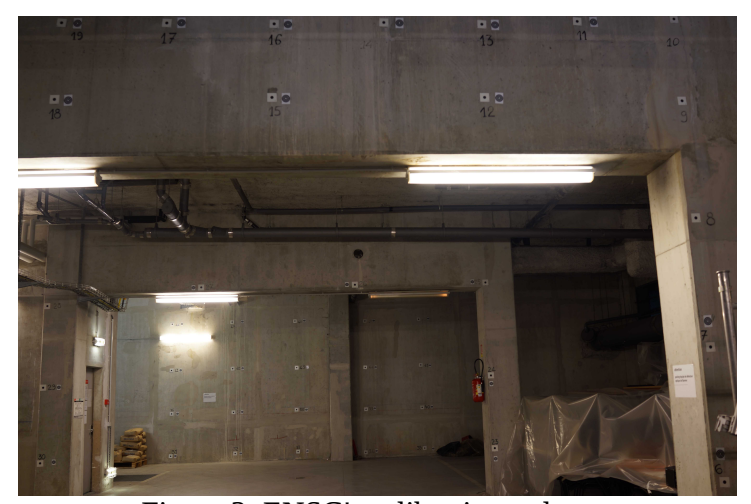

Figure 3: ENSG's calibration polygon

We first calibrated the camera on a bench specially set for that purpose (see Fig. 3), on which the references have been determined with a submillimetric accuracy, using metrological 
methods. We then refined this model on our UAV pictures (auto-calibration using the homologous points), and the process ended in delivering a visually good 3D model. Table 1 details the Mean Absolute Error (MAE) and the Root Mean Square Error (RMSE) of the residuals after the compensation on the 50 GCP.

\begin{tabular}{|l|c|c|c|c|c|}
\cline { 2 - 6 } \multicolumn{1}{c|}{} & X & Y & Z & XY & XYZ \\
\hline MAE $(\mathrm{mm})$ & 8 & 6 & 18 & 11 & 23 \\
\hline RMSE $(\mathrm{mm})$ & 10 & 7 & 23 & 12 & 26 \\
\hline
\end{tabular}

Table 1: Indicators on the residuals after the compensation

When we interpolated the computed model at the GCP emplacements, we observed a sort of bending on the extremities of the site. Figure 4 shows that this bending effect can bring errors over $10 \mathrm{~cm}$ on the edges.

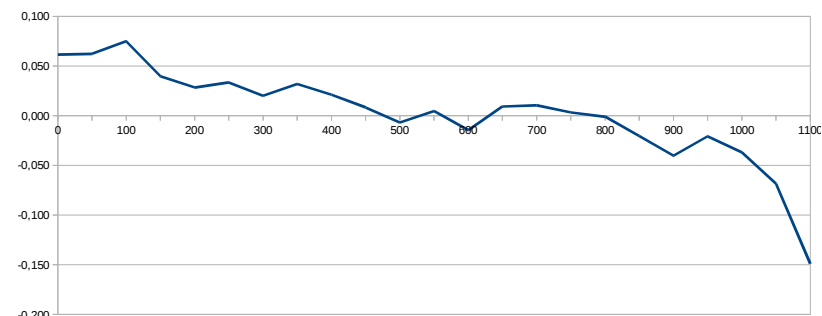

Figure 4: Differences in height (m) alongside the dyke, between the interpolated DTM and the levelling coordinates

\section{FURTHER WORK ON ROCHEMAURE'S DYKE}

\subsection{Terrestrial Lidar survey}

Rochemaure's dyke has been used as a test site for an accuracy assessment on terrestrial Lidar survey, aiming to determine wether laser scanner is a fitting instrument to monitor dykes. In April 2013, two independent surveys have been conducted, moving the references between each operation to be sure surveys are not linked in any way. A Riegl LMS620 scanner has been used, which is a professional equipment costing over $120000 €$. It took 6 days of work and two engineers to complete both surveys of the $60 x 600 \mathrm{~m}$ dyke. Each scan is actually the result of 39 stations, merged using 5 to 12 references, with an average RMSE of $10 \mathrm{~mm}$.

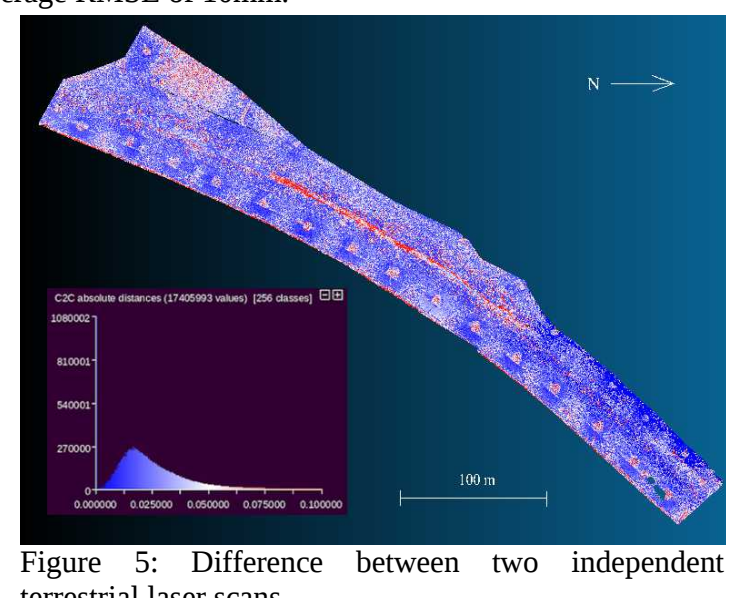

terrestrial laser scans

We compared the resulting point clouds using CloudCompare, which computes the Haussdorf distance between two point clouds (Girardeau-Montaut 2006). Figure 3 shows the differences ranging from 0 to $10 \mathrm{~cm}$ between both determinations. This comparison follows a Gaussian distribution with the following parameters :

- Mean absolute error : 23mm

- $\quad$ Standard deviation : $16 \mathrm{~mm}$

We can observe four zones presenting high differences :

- Around the scanning station on the main road

- Along the subsidiary road on the west (a thin red strip)

- At the eastern extremity, along the river

- $\quad$ On the south east bushes

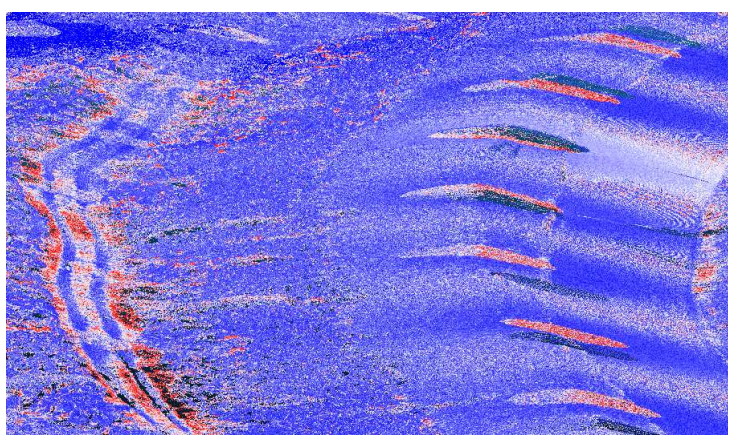

Figure 6: Zoom on some imprecisions between surveys Errors on the most vegetalized zones are easily understandable, as well as the ones on the extrimity along the river which required some grazing angles from the scanner.

As Figure 5 shows, some of the inaccuracies can be correlated with the lowest densities. The comparison process used by CloudCompare, can explain this result. Another grid to grid comparison has been computed with Surfer, and another mesh to mesh comparison with Polyworks. On these, the area around each scanning station did not show abnormally high residuals. Indeed, as it is not possible to scan directly under the equipment, the points density were lower than 100 points per square meter, which represents one point each $10 \mathrm{~cm}$ on a $1 \mathrm{~m}$ square. Computing the absolute distances to these references logically shows some imperfections.

However, we still had this red line on the west part, which is actually a subsidiary road, in which a small leak in the dyke caused puddles. This zone might have changed a bit between both surveys, but it is also complicated to finely measure this environment.

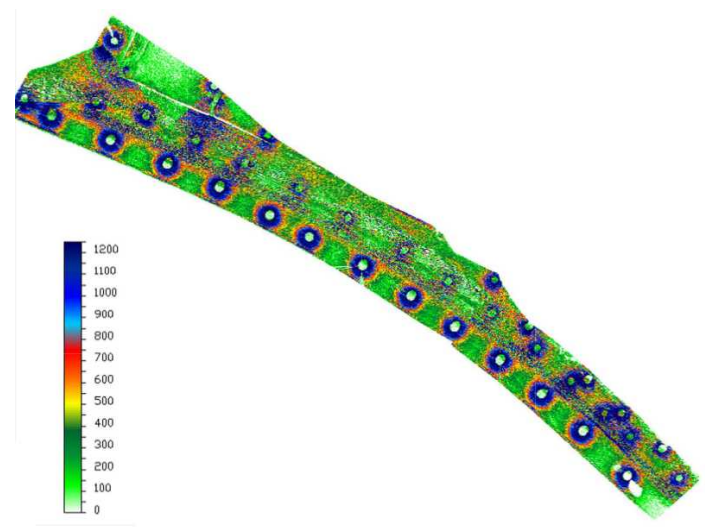

Figure 7: Density of points per square meter

\subsection{Photogrammetric processing}

End of june 2013, 2 months after the Lidar mission, a photogrammetric survey campaign had been organized on Rochemaure's dyke. Similarly to La Pallière, we set up two rows of GCP on a 50 meters grid, surveyed them both by GPS and precision levelling. We then used IGN's HexaXL UAV to 
acquire our pictures with an $80 \%$ overlap on two parallel strips, at 60 meters high to get centimetric pixels.

Although the UAV survey was fast this time (two hours between the first and the last picture), as we knew the hardware and learnt from our mistakes, we actually had to postpone the flight due to the particularly windy conditions. That is one of the UAV's drawback, and this underlines that using "low cost" material as the HexaXL instead of a heavier equipment could cause problems to a company relying only on this. In these cases, microlight systems could be a solution.

The acquisition a couple of days later had however been successful, 110 pictures have been taken, on which we ran different tests.

\subsubsection{Distortion models tested}

The Apero tool -part of the MicMac suite- has been used to compute bundle adjustments. It natively includes different distortion models; we used the following ones :

- "RadialBasic" : model with 6 degrees of freedom (1 for the focal length, 2 for PPA and PPS, 3 coefficients for the polynomial correction $-r^{3}, r^{5}$ and $r^{7}$ )

- "RadialStandard" : evolution of RadialBasic in which the center of distortion and the principal point are not constrained to be equal any more (8 degrees of freedom)

- "RadialExtended" : evolution of RadialStandard in which we add two extra coefficients to the polynomial correction $-r^{9}$ and $r^{11}$ (10 degrees of freedom)

- "FraserBasic" : model composed 10 degree of freedom (focal length, PPAS and PPS supposed equal, 3 coefficients for radial correction, an affine and a decentric part)

- "Fraser" : evolution of the FraserBasic in which the center of distortion and the principal point are not equal any more (12 degrees of freedom)

Each of these models are used in classical photogrammetry. Fraser models are described in (Fraser 1997), and the interested reader can also find formulas in the software's documentation.

\subsubsection{Bundle adjustment residuals}

We refined the existing ENSG calibration polygon by adding new targets. With the help of the last year student from ENSG, these have been determined from 5 different stations. Using IGN's Comp3d calculation software, we were able to get coordinates with a submillimetric accuracy.

From a set of 8 pictures made with various angles of view, we have computed 4 calibration models. The mean absolute errors of these residuals are listed in Table 2. Note that we used the same image measures for each computation.

\begin{tabular}{|c|c|c|c|c|}
\hline & $X(\mathrm{~mm})$ & $Y(\mathrm{~mm})$ & $Z(\mathrm{~mm})$ & Image (pixel) \\
\hline RadialBasic & 12 & 5 & 3 & 0,64 \\
\hline RadialStd & 4 & 1 & 1 & 0,38 \\
\hline RadialExtended & 10 & 3 & 2 & 0,51 \\
\hline FraserBasic & 9 & 3 & 2 & 0,50 \\
\hline Fraser & 9 & 3 & 2 & 0,50 \\
\hline
\end{tabular}

Table 2: MAE on the GCP during the calibration process

Using the RadialStd model we get the best residuals, while the RadialBasic does not seem to be very adapted to the situation. On the other way, the most complex models give residuals between these.

We then used these calibration models on our UAV pictures, and computed the bundle adjustments following two different strategies :

- "Figee" : Freeze the calibration parameters in the bundle adjustment
- "AutoCal” : Re-evalutes the calibration parameters, which are released in the bundle adjustment.

For the AutoCal process, we tried to firstly adapt it on a central subsample from our dataset - 20 pictures at the middle of the dyke - and then used that new calibration on the entire dataset. That step finaly proved to be pointless, as we obtained similar results while working directly on the full dataset. Table 3 lists the different results we had for these models.

It seems to indicate that the calibration made on the polygon should not remain frozen, although we noticed some slight improvement while using Figee with the FraserBasic model.

The 5 coefficients distortion model applied on RadialExtended leads to the best residuals, while adding an affine and a decentric part (Fraser) leads to the worst one.

\begin{tabular}{|c|c|c|c|}
\cline { 2 - 4 } \multicolumn{1}{c|}{} & Figee & AutoCal & Evolution \\
\hline RadialBasic & 0,951 & 0,939 & $+1,26 \%$ \\
\hline RadialStd & 0,990 & 0,936 & $+5,45 \%$ \\
\hline RadialExtended & 0,778 & 0,769 & $+1,16 \%$ \\
\hline FraserBasic & 0,935 & 0,938 & $-0,32 \%$ \\
\hline Fraser & 0,998 & 0,935 & $+6,01 \%$ \\
\hline
\end{tabular}

Table 3: Average residuals from the bundle adjustment in different scenarios

Considering the center of distortion and the principal point to be equal or not does not seem to have a big effect on the solution. Although, as shows Table 4 , the computed values can be very different on our 4000 by 6000 pixels images.

\begin{tabular}{|c|c|c|}
\cline { 2 - 3 } \multicolumn{1}{c|}{} & $d X$ & $d Y$ \\
\hline RadialStd & 15,75 & 25,66 \\
\hline RadialExtended & 19,60 & 15,46 \\
\hline Fraser & 5,66 & 93,56 \\
\hline
\end{tabular}

Table 4: Difference (in pixels) between the principal point and the center of distortion

We tried to get even better results from the RadialExtended model by applying a two steps individual calibration process. The first one consists in releasing the focal length as an unknown during the bundle adjustment process, so that for each image it computes an optimal value minimizing the residuals. Fig 7 shows the evolution of that focal length (in pixels), compared to its average value.

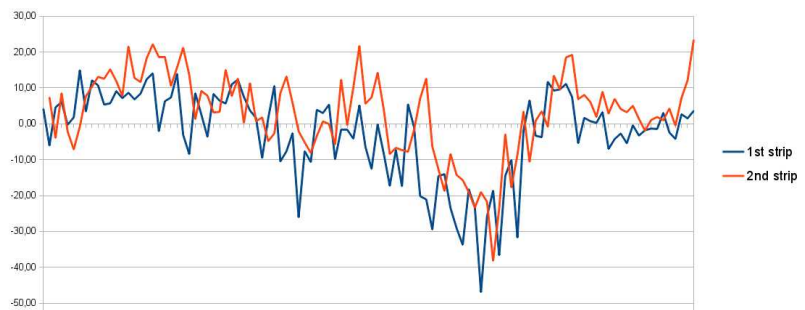

Figure 8: Difference between each individual focal length and the average value (in pixels)

The evolution of the focal length on both strips is similar, which is comprehensible as the images have an overlap of $80 \%$ and so share most of their tie points. The difference between the highest and the lowest value reaches 60 pixels, which represents $1 \%$ of the mean value. This first step ended with an average residual of 0,768 pixels.

The second step consists in releasing the five parameters of the distortion model, as well as the principal points coordinates. With the previous focal length associated and now fixed, we can now compute for each image an optimal calibration model. This second bundle adjustment ended with an average residual of 0,767 which is very similar to the residuals we obtained with the AutoCal option. 
The calibration per image process does not seem to improve much the solution, although some differences are noticeable in the principal points, distortion centers and parameters asociated to each picture.

\subsubsection{Residuals on the GCP (compensation process)}

Likewise in La Pallière, we equipped the site with 2 parallel strips of ground control points, so every 50 meters we had one GCP at both extremities of the dyke. Using all these points in the compensation process, we obtained the residuals listed in Table 5.

\begin{tabular}{|c|c|c|c|c|c|c|}
\hline & $\bar{X}$ & $Y$ & $Z$ & $X Y$ & $X Y Z$ & \\
\hline \multirow{2}{*}{$\begin{array}{c}\text { FraserBasic } \\
\text { AutoCal }\end{array}$} & 13 & 11 & 31 & 17 & 35 & $M A E(m m)$ \\
\hline & 16 & 13 & 39 & 21 & 44 & RMSE (mm) \\
\hline \multirow{2}{*}{$\begin{array}{c}\text { Fraser } \\
\text { AutoCal }\end{array}$} & 12 & 10 & 35 & 17 & 41 & MAE (mm) \\
\hline & 15 & 12 & 44 & 19 & 48 & RMSE (mm) \\
\hline \multirow{2}{*}{$\begin{array}{c}\text { RadialBasic } \\
\text { AutoCal }\end{array}$} & 10 & 7 & 20 & 14 & 26 & $M A E(m m)$ \\
\hline & 12 & 9 & 27 & 15 & 31 & RMSE (mm) \\
\hline \multirow{2}{*}{$\begin{array}{l}\text { RadialStd } \\
\text { AutoCal }\end{array}$} & 18 & 10 & 23 & 22 & 35 & $M A E(\mathrm{~mm})$ \\
\hline & 20 & 12 & 30 & 23 & 38 & $R M S E(\mathrm{~mm})$ \\
\hline \multirow{2}{*}{$\begin{array}{c}\text { RadialExtended } \\
\text { AutoCal }\end{array}$} & 8 & 6 & 18 & 11 & 23 & $M A E(\mathrm{~mm})$ \\
\hline & 10 & 7 & 23 & 12 & 26 & $R M S E(\mathrm{~mm})$ \\
\hline \multirow{2}{*}{$\begin{array}{l}\text { RadialExtended } \\
\text { Figee }\end{array}$} & 7 & 6 & 19 & 10 & 23 & $M A E(\mathrm{~mm})$ \\
\hline & 8 & 7 & 24 & 11 & 26 & $R M S E(\mathrm{~mm})$ \\
\hline \multirow{2}{*}{$\begin{array}{l}\text { Calibration } \\
\text { Per Image }\end{array}$} & 8 & 6 & 19 & 11 & 24 & $\operatorname{MAE}(\mathrm{mm})$ \\
\hline & 10 & 7 & 23 & 12 & 26 & $R M S E(\mathrm{~mm})$ \\
\hline
\end{tabular}

Table 5: Indicators on the residuals (mm) after compensation on the GCP

As the table shows, it is now the Fraser model that gives the worst results. The RadialExtended model still gives the best ones. We can notice that the AutoCal, Figee, or Calibration Per Image end in with the same kind of results. Only 1 millimeter on the Z-axis can distinguish themselves, and at this the AutoCal model gives very slightly better results.

\subsubsection{Comparison with Lidar}

We have seen that terrestrial Lidar can acquire high quality data, and can be used to monitor centimetric deformations. However that equipment is expensive, and as it is heavy it can not be embedded on UAV so far, although some developments have been successful (Conte 2013). It is however not possible to monitor centimetric deformations that way, and thus can not be used in an emergency situation on a dyke.

We were interested in confronting these terrestrial surveys to the different models that we generated with MicMac from the previous results.

Table 6 lists the results of the comparison between each model computed from the previous orientations and both Lidar surveys.

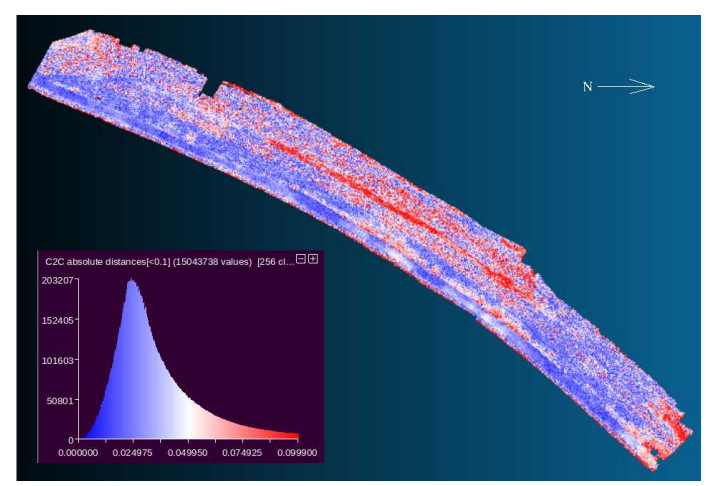

Figure 9: Comparison between the first Lidar survey and the pointcloud from the RadialBasic model

\begin{tabular}{|c|c|c|c|}
\hline & & Lidar 1 & Lidar 2 \\
\hline FraserBasic & Mean & $41 \mathrm{~mm}$ & $40 \mathrm{~mm}$ \\
\hline AutoCal & Sigma : & $22 \mathrm{~mm}$ & $21 \mathrm{~mm}$ \\
\hline Fraser & Mean : & $38 \mathrm{~mm}$ & $38 \mathrm{~mm}$ \\
\hline AutoCal & Sigma : & $21 \mathrm{~mm}$ & $21 \mathrm{~mm}$ \\
\hline RadialBasic & Mean : & $34 \mathrm{~mm}$ & $35 \mathrm{~mm}$ \\
\hline AutoCal & Sigma : & $18 \mathrm{~mm}$ & $18 \mathrm{~mm}$ \\
\hline RadialStd & Mean : & $37 \mathrm{~mm}$ & $36 \mathrm{~mm}$ \\
\hline AutoCal & Sigma : & $19 \mathrm{~mm}$ & $19 \mathrm{~mm}$ \\
\hline RadialExtended & Mean : & $35 \mathrm{~mm}$ & $34 \mathrm{~mm}$ \\
\hline AutoCal & Sigma : & $18 \mathrm{~mm}$ & $18 \mathrm{~mm}$ \\
\hline RadialExtended & Mean : & $38 \mathrm{~mm}$ & $37 \mathrm{~mm}$ \\
\hline Figee & Sigma : & $20 \mathrm{~mm}$ & $20 \mathrm{~mm}$ \\
\hline Calibration & Mean : & $41 \mathrm{~mm}$ & $36 \mathrm{~mm}$ \\
\hline Per Image & Sigma : & $22 \mathrm{~mm}$ & $20 \mathrm{~mm}$ \\
\hline
\end{tabular}

Table 6: Average residuals from the bundle adjustment in different scenarios

The average results are similar if we compare our work to the first Lidar survey or to the second one. The absolute distances follow a gaussian distribution globally centered around $4 \mathrm{~cm}$ with a standard deviation of $2 \mathrm{~cm}$, independently of the model we use. This precision is sufficient for the exceptionnal acquisition case, but should be improved if we want to use photogrammetry from UAV for the regular monitoring.

Although we previously noticed in Table 2 and Table 3 that the residuals were the higher while using the RadialBasic model, the comparison between the point cloud generated from and the Lidar surveys give the better results. The RadialExtended model, which gave the best results in Table 2 and 3, also shows here similar results.

The Figee mode, as well as the individual calibration process, both applied on the RadialExtended model, did not improve these.

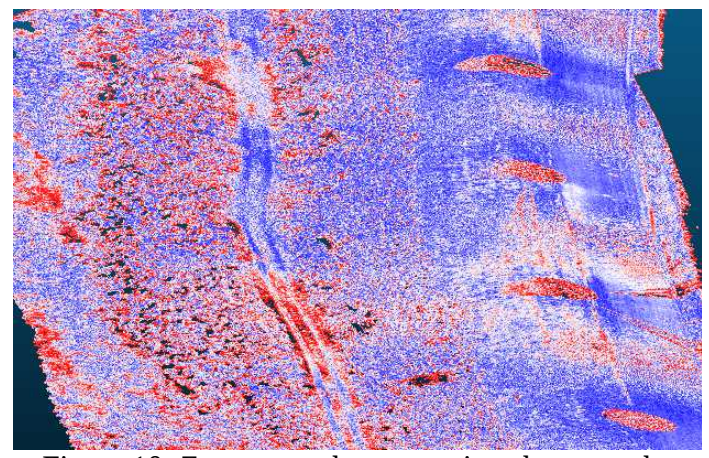

Figure 10: Zoom on a the comparison between the FraserBasic model and the first Lidar survey

We can see on them the same kind of bad interpolation around the laser scanning stations, which seems logical as the point density is lower. We do not get the same results using the radial distorsion models, but only with these : Fraser, FraserBasic, RadialExtended Figee, Individual calibration per image.

The imprecisions around the puddles are still present, but more subtlety than on the first comparison. Actually, the entire zone on the north presents high differences to the Lidar survey. That zone being vegetalized, it is not very surprising to have such differences in our comparisons, two months separating both surveys.

These results are disturbing as we do not get what we expected at the beginning. We thought that calibrating the camera on a special bench set for that purpose, by using a complex distortion model would end in better results. As the RadialBasic and RadialExtended comparisons show, it did not.

We did not find any correlation between the calibration residuals, the bundle adjustment results, or the residuals on the 
GCP when we compared the issued model with the Lidar surveys.

\section{FURTHER WORK}

Our previously presented work showed that minimizing the average residuals from the bundle adjustment is not sufficient to deliver the best 3D model. However, refining the position, the orientation, as well as the distortion model seems to be the key to improve the quality of our work. Analyzing the results of the different bundle adjustments is hard as we do not have any ground truth, except from the GCP. To this date, embedded GPS and IMU are not accurate enough to get a precise indicator of the position and orientation of the camera.

Thanks to the École Supérieure des Géomètres et Topographes (ESGT), we have been able to simulate a UAV flight at a 1/20 scale. By fixing a camera on a structure to get constant orientations, we took pictures of the facing wall (supposed to be the dyke) - textured with maps for the tie point detection to be efficient. The camera was sliding on a "perfectly straight" rail (supposed to be the UAV), on which we measured the displacements between each pictures.

To simulate faithfully the conditions required on the field, the accuracy of the GCP had to be improved from the same rate. We set up on the wall a network of 75 targets that we surveyed with metrological methods. Each target has been measured 8 to 14 times, resulting on RMSE around $0,2 \mathrm{~mm}$. The position of the camera has been measured using a Renishaw XL-80 interferometer, assuring a $0,5 \mathrm{ppm}$ accuracy. Navigating between 0 and 50 meters, the accuracy on the positions should not exceed 25 microns.

We hope to be able to use these data to get a clear idea on how the bundle adjustment results influence the position and orientation results. We believe that the tie points detection applied on the textured wall delivered many tie points, but their quality and repartition could be improved to approach the centimetric accuracy required.

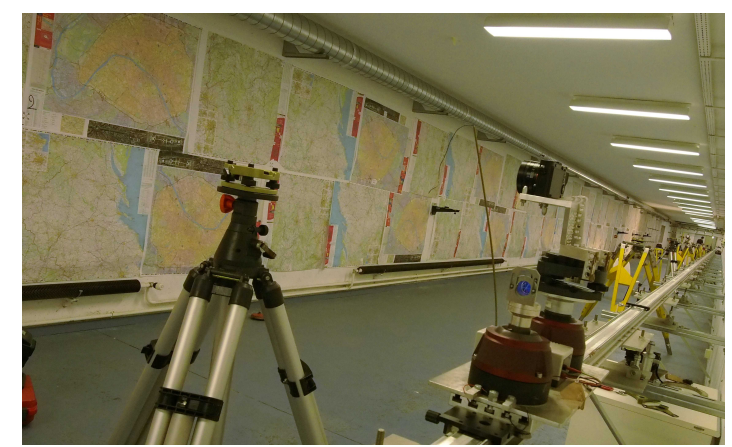

Figure 11: Simulation of an under-scaled UAV flight in a controlled environment

\section{CONCLUSION}

We saw in the first part that UAV can be used to acquire high quality pictures easily, although some adjustments are often needed. We believed that the residuals we obtained in the bundle adjustment or during the compensation could give an idea on the quality of the issued 3D model.

A comparison with terrestrial Lidar surveys showed that these are not to be trusted, as some of the worst bundle adjustment residuals actually delivered the best results. However, minimizing these residuals (on a same model) by differents techniques leads to better results.

The technology has however proved to be already efficient when a limited accuracy is sufficient. It seems possible with the actual technology to detect some of the $5 \mathrm{~cm}$ deformations as required by CNR in case of an exceptional situation.
Some further work on a controlled environment could help us to improve that accuracy, and possibly to fulfill CNR's dykes monitoring needs.

\section{REFERENCES}

Conte, G., Kleiner, A., Rudol, P., Korwel, K., Wzorek, M., Doherty, P., 2013. Performance evaluation of a light-weight multi-echo Lidar for unmanned rotorcraft applications. IAPRS Volume XL-1/W2, pp 87-92, 4-6 September 2013, Rostock, Germany.

Cramer, M., Bover, S., Gütlinger, M., Honkavaara, E., McGill, A., Rijsdijk, M., Tabor, M., Tournadre, V., 2013. On the use of RPAS in national mapping - the EuroSDR point of view. IAPRS Volume XL-1/W2, pp 93-99, 4-6 September 2013, Rostock, Germany.

Fraser, C., 1997. Digital camera self-calibration, ISPRS Journal of Photogrammetry and Remote Sensing, vol. 52, issue 4, pp. 149-159.

Girardeau-Montaut, D., 2006. Détection de changement sur des données géométriques. Thesis manuscript, Telecom Paris.

Kasser, M., Egels, Y., 2002. Digital photogrammetry. Taylor \& Francis, London.

Pierrot-Deseilligny, M., Cléry, I., 2011. APERO, an open source bundle adjustment software for automatic calibration and orientation of a set of images. Proceedings of the ISPRS Commission V Symposium, Image Engineering and Vision Metrology, 2-4 March 2011, Trento, Italy.

Pierrot-Deseilligny, M., Paparoditis, N., 2006. A multiresolution and optimization-based imagematching approach : An application to surface reconstruction from SPOT5-HRS stereoimagery. IAPRS vol XXXVI-1/W41, February 2006, Ankara, Turkey.

Remondino, F., Fraser, C., 2006. Digital camera calibration methods, considerations and comparisons. IAPRS Volume XXXVI, Part 5, 25-27 September 2006, Dresden, Germany

Robin, Y., 2013. Lever lasergrammétrique terrestre d'une portion de digue. Internal report.

Stempfhuber, W., 2013. 3D-RTK capability of single GNSS receivers. IAPRS Volume XL-1/W2, pp 379-384, 4-6 September 2013, Rostock, Germany.

\section{References from internet}

Piksi : http://swift-nav.com/piksi.html, last access October 30th 2013. 